\title{
VOCABULAIRE DES NOMS DE PLANTES MEDICINALES ET ALIMENTAIRES \\ Utilisées par les Mélanésiens de Lifou (lles Loyalty)
}

\author{
Par Maurice H. LENORMAND (1).
}

J'exprime mon souvenir reconnaissant au regretté Professeur BAAS-BECKING, ancien Directeur du Jardin de Buitenzorg (aujourd'hui Johore) à Java, puis conseiller de recherches à la Commission du Pacifique Sud à Nouméa, qui vérifia l'identification botanique d'un certain nombre de plantes, dans les débuts de ce travail.

Je remercie tout spécialement $M$. SснмıD, chef du département de Botanique à l'Institut Français d'Océanie (O.R.S.T.O.M.) qui a apporté le concours indispensable de ses connaissances en systématique dans le travail de recoupement et d'identification fait en collaboration et qui nous a permis d'établir ensemble une première liste d'environ 220 noms vernaculaires correspondant à peu près à la plupart des désignations connues par le commun des habitants de Lifou.

Le présent vocabulaire, qui représente plusieurs années de travail évidemment intermittent, comprend environ 500 noms de plantes, dont une dizaine sont donnés sans identification botanique. Une cinquantaine de plantes collectées et citées en publication par les botanistes qui m'ont précédé dans l'étude de la flore de Lifou, n'ont pas reçu place dans ce vocabulaire, leur désignation en langue de Lifou n'ayant pas encore pu être recueillie.

J'exprime ma profonde et sincère gratitude à mes principaux informateurs Lifou : le chef de tribu Hnoija Wahnyamala, oncle de ma femme, la guérisseuse Louise Hnasaïé et le guérisseur Picié du village de Kejëny.

La plupart des noms vernaculaires donnés en français sont les noms locaux utilisés par les habitants de la Nouvelle-Calédonie. Les Européens ont créé une terminologie des noms des plantes

(1) Pharmacien de la Faculté de Paris. Lauréat des Langues Orientales. 
qu'ils ont trouvées en se servant des vocables français, mais en les appliquant à des espèces végétales voisines ou n'ayant parfois qu'une ressemblance très approximative avec la plante européenne de même nom; ainsi, pour baume, lilas, cerisier, prunier. Ceci explique également l'usage abondant du qualificatif « faux» comme correctif dans les désignations pratiquées par le parler français local, par exemple: fausse pistache, faux topinambour, fausse citronnelle, fausse ramie, faux chêne blanc et même faux gaïac, faux santal.

A côté des noms calédoniens français, les noms anglais ont souvent été indiqués ètant plus largement connus et utilisés pour la désignation courante des espèces appartenant à la région botanique de l'Indo-Pacifique.

La lecture des mots lifou pourra sembler assez déroutante et rébarbative. ("est l'orthographe traditionnelle et habituelle selon laquelle la langue est écrite qui a été utilisée et cela pour plusieurs raisons. Disons tout d'abord que l'utilisation d'un des systèmes internationaux de notation phonétique ne rend pas plus aisé le déchifrrement des vocables si le lecteur n'est pas un linguiste; au surplus, du point de vue de la notation graphique, le système adopté pour le lifou est, du point de vue phonématique, un système simple, correct et cohérent. Une autre raison de son utilisation est que la transcription des mots dans l'orthographe ordinaire des gens de Lifou permet à ceux-ci et à toute autre personne qui peut lire cette langue, de reconnaitre parfaitement les mots et de les reproduire oralement avec une prononciation correcte qui les rend aisément compréhensibles. Le lifou possède 12 consonnes et une voyelle qui n'existent pas en français; la meilleure solution nous a paru dans le choix de l'orthographe courante de la langue.

Afin de permettre au lecteur non initié de connaître la réalisation des phonèmes lifou ne coïncidant pas avec les sons qu'expriment les lettres de l'alphabet français, nous indiquerons ci-après, la valeur des caractères alphabétiques particuliers au Lifou. Ce sont ë, ö, u, dr, tr, j, th, x, q, ny, ng, hI, hm, hn, hny, hng.

Ia voyelle ë est un è très ouvert, plus ouvert encore qu'en français dans « air ».

La voyelle ö correspond au son « oe $»$ dans \& wuvre».

La voyelle u se prononce toujours * ou \& conme dans \& cou».

c se prononce teh comme dans « tchèque» ainsi qu'en italien dans « eitta».

dr et tr se prononeent à peu près comme en anglais dans * dream * et dans « try $»$. 
th se prononce comme en anglais dans * though » tandis que $j$ (qui devrait logiquement s'écrire $d h$ ) se prononce comme en anglais dans « mother».

$\mathrm{X}$ correspond au $\mathrm{X}$ grec et à la jota espagnole.

q correspond à peu près au « wh » anglais dans what?

ny correspond au ñ mouillé dans canyon.

ng se prononce d'une seule émission de voix, à peu près comme dans le mot anglais « singing ».

Enfin le lifou, parallèlement aux consonnes I, m, n, ny, ng, sonores possède une séric de phonèmes non sonores qui se réalise avec une aspiration ou plus exactement une expiration. Ils sont orthographiés hl, hm, hn, hny, hng.

Aelan

Agava (Lôsi)

Ageti temoni, Ageti

tepolo, of. Xothahao Agoze

$A i$, cf. Panganai

Ailope

$\boldsymbol{A j u}$

Akötra
Al, ef. Xaca

Alu

Aluxen

Amane

Apinyiwa (Wet)

$=\operatorname{Agava}($ Lösi)

Atremele kapalulu n. d'un clone de Dioscorea alata, igname. Dioscoréacées.

var. Agawa wië, Agawa madra. Macaranga vedeliana Mull. Arg. (= Acalypha vedeliana Baill.). Euphorbiacées.

Acacia farnesiana Dän, Légumineuses, cassie.

Strobilopanax macrocarpus R. Vig. $(=$ Meryta macrocarpa Baill. Araliacées.

Geilonoplesinm cymosum (R. Br.) A. Lum. Liliacées, asperge canaque.

Malvestrum tricuspidalum Asa Gray, Malvacées.

Aju madra. Planchonella lifuensis Pierre, Sapotacées, azou rouge.

Aju wië. Planchonella ligyensis Piettr (=P. microphylla), Sapotacées, azou blanc.

Colocasia sp. Aracacées -- taro sauvage.

Premna integrifolia Mant. (=P. sambucina L.). Verbenacées, sureau de Nouvelle-Calédonie, alagaó de Malaisic.

Pueraria thumbergiana Benth. (= P. neo Calëdonica Harms = Pacchyrhizus thumbergianus Sieb. et Zuce.). Légumineuses, magnagnia. var. plus comestible de Pneraria thumbergiana. clone de Colocasium antiquorum, taro. Aracacées. Macaranga vedeliana Mull. Arg. Euphorbiacées.

Graphtophyllum piclum. Acanthacées, sarása de Malaisic. 
Atremen

Atrejop

Atulet

Awo

Awahao

Axö

Axöti ne hnit, cf. otr.

Belep
Ben
Bili
Bona, cf. Naina

Bui

Bula

Caina

Canea

Canehmez

Canevexipo

\section{Cangen}

Cawe

Cica

Cijeni

Cila (wet) ef. Haoca (Lösi)

\section{Cing}

Co

Drelë

Dreli

Drem
Laportea photoniphylla Wedd. Urticacées, nangalat des Nouvelles-Hébrides.

clone de Saccharum officinarum, canne à sucre.

Teucrium villosum SW. Labiées.

Bambusae. Graminées, bambou.

Ficus obliqua Forst. (=F. aphanoneura Warb.). Moracées, banyan blanc.

Ficus scabra Forst. var. pubescens $(=$ F. storckii Seem. var. Warb. pubescens. Moracées.

Pseudomorus brunoniana Bur. Moracées.

n. d'un clone de Dioscorea alata, igname.

n. d'un clone de Dioscorea alata, igname.

Ocimum basilicum L. Labiées, basilic.

Cajanus pseudocajan Schnz. $(=C$. indicus

Spreng.). Légumineuses, ambrevade, pois d'Angole.

clone de Colocasium antiquorum, taro.

clone de Colocasium antiquorum, taro.

clone de Musa sapientium, bananier.

Pavetta opulina D.C. Rubiacées.

Tylophora affine tapeinogyne. Asclépiadacées.

Momordica charentia L. Cucurbitacées, amargoso, margose.

Alectryon carinatum Radlek. Sapindacées.

nom d'un clone de Musa sapientium, bananier.

Excoecaria agallocha L. Euphorbiacées, palétuvier blane.

n. d'un clone de Dioscorea alata, igname.

Hemicyclia deplanchei Baill. Euphorbiacées.

Bruguiera eriopetala Stendel. Rhizophoracées, palétuvier rouge.

n. d'un clone de Musa sapientum, bananier.

Erythrina variegata $(=E$. glauca Willd. var. fastigiata Guill.). Légumineuses, erythrine, peuplier canaque.

Ipomoea pes-caprae Roth. Convolvulacées, piedde-chèvre.

Breynia disticha Forst. var. neo caledonica. Euphorbiacées. 
Dremixapo

Dridrem

Drön

Dru

Egösae ë

Ekenej.

Eköhaitr

Eköqadri

Eku

Elehao

Elu

Emëzi

Enaji

Eno

Eo

$\boldsymbol{E}_{\boldsymbol{p}}$

Epeth

Epiatr.

Eteteij.

Etha

Ethahao

$\boldsymbol{E u j}$

Fafino

Faimate, cf. Wacua

Faio

Falaboia
Adenanthera pavonina L. Légumineuses, coral beadtree, sága de Malaisie.

Xylosima lifuana. Flacourtiacées.

Nicotiana fragrans Hooker $(=N$. suaveolens

Lehm. Nicot). Solanacées, tahac.

Nicotiana fragrans Hooker $(=N$. suaveolens

Lehm. Nicot). Solanacées, tabac (feuilles).

Peperomia leptostachya Hook et Arn.

Peperomia baueriana Miq.

Peperomia endlicheri.

Peperomia insularum. Piperacées.

Geniostoma sarasini Dän. Logoniacées.

Syzygium pseudo-pinnatum I. $(=S$. verrucosum Dän.). Myrtacées.

Cardiospermum halicabium L. Sapindacées.

Cycasia circinalis L. Cycadacées, cycas.

Geodorum pictum Lindl. Orchidacées.

Hibiscus tiliaceus L. Malvacées, bourao.

Hypserpa neo-caledonica Diels. Menispermacées.

Lycopodium phlegmaria. Lycopodiacées; cheveux de sorcière, galons de gendarme, barbe de rat.

Fourcroya gigantea. Amaryllidacées, dit aloès en Nouvellè-Calédonie.

Oxera balansae. Verbenacées.

Hernandia cordigera Vieill. Hernandiacées, boisbleu.

Hernandia ovigera Sol. Hernandiacées, bois-bleu. Psychotria bailloni Schlechter. Rubiacées.

Casuarina equisetifolia Forst. Casuarinacées, boisde-fer.

Ficus virigata $\mathrm{RWV}$ (=F. philippinensis Bur.) var. sessilis.

- 1. équivalent de Awahao, Ficus obliqua.

- 2. Vitex trifoliata L. f. Verbenacées.

Guioa fusca. Sapindacées.

Cirossotyles biflora. Rhizophoracécs, hêtre noir noueux.

Ficus edulis. Moracées, figuier à figue rouge.

Phaseolus vulgaris. Légumineuses, haricot.

Delonix regia (= Poinciana regis Boj.). Légumineuses, flamboyant. 
Falanipana

Famuken

\section{Fenij}

Fenije ne helep

Fetramit

Fetraaicatei

cf. Fetraxeci

Fetra hana

\section{Fetrahao}

Fetranië, cf. Fetrahao

Fetraxaji, Fetraalaxaji

Fetraxeci, ef. Fetra ai catei

Ficahlu

\section{Finyiqat \\ Fijuë \\ Fiket \\ Fit, ifit \\ Fufuce \\ Fugöt \\ Fuo}

\section{Gaae}

Gaga

Ge

Gem. (Lôsi),

cf. Kuka (Wet)

Gemegem

\section{Gili}

Goana

Gölep (Lôsi)
Plumeria rubra Ait. Apocynacées, frangipanier.

Passiflora edulis Sims. Passifloracées, pommeliane, passion-fruit, grenadille, passiflore.

Fenije madra

Fenije wië

Lofoschaenus arundinaceus Forst. Cypéracées.

Cleidion verticillatum Baill. Euphorbiacées.

Psychotria nummularioides Guill. Rubiacées.

Cleidion vieillardii var. macrophyllum Baill. Euphorbiacées.

Hybanthus ilicifolius Schinz. $(=H$. caledonicus Guill.). Violacées.

Hybanthus ilicifolius Schinz. Violacées.

Psychotria nummularioides Guill. Rubiacées.

Evartmia orientalis (= Tabernae-montana orientalis $\mathrm{R}$. Br.). A pocynacées.

Andropogon contortus L. Graminées.

Lippia nodiflora Rich. Verbenacées.

Calophyllum inophyllum L. Guttiferacées, Alexandrian Laurel, tamanou.

n. d'un clone de Dioscorea alata, igname.

n. d'un clone de Dioscorea alata, igname à cochon.

- 1. Citrullus vulgaris Schrad, citrouille.

- 2. Cucurbita pepo L., courge, potiron, Cucurbitacées.

Elaeocarpus rotundifolius Brong. et Gris. Elacocarpacées, cerisier de forêt.

Morinda candollei var. villosa Guill. Rubiacées.

Lagenaria vulgaris Set. Cucurbitacées, gourde.

Mucuna gigantea (Willd.) D.C. Légumineuses, haricot géant (= Dolichos giganteus Willd.).

Caesalpinia nuga Ait. Hort. Légumineuses, accroche-coxur. Dápinit des malais.

n. d'un clone de Dioscorea alata, igname.

n. d'un clone de Dioscorea alata, igname.

Octotheca ouveana (Dän.) Guill. (= Dizygotheca ouveana Dän.). Araliacées, golip rouge. 
Gölep (wet)

Gorok

Gäti

Guafa

Gufetren

Gulago

Gum

\section{Gusupen}

Gutu

Gutuma

Hae

Haetraqa

Hagè

\section{Hainy}

Halo sinoë, cf. Pahatre ne sinoë

Ham

Hane ura

Hanyimu ne gejë

Hanyimu ne helep

Haoca (Lôsi), cf. Cila (Wet)

Hao ne gejë

Hao papale

Hao puny

Hao thotho

Hapetra
Schlefera golip Baill. Araliacées, golip blanc à pétiole vert, ralia.

Capsicum frutescens L. Solanacées, piment.

Araucaria cooki $\mathrm{R}$. Br. Conifères, pin colonnaire.

Psidium guayava L. Myrtacées, goyavier.

n. d'un clone de Dioscorea alata, igname.

Peziza auricularia. Champignons, pezize, oreillesde-sorcière.

Gumi papale. Ricinus communis L. Euphorbiacées, ricin.

Gumi ne wet. Aleurites moluccana (L.) Wildnow. Euphorbiacées, noyer de bancoule, bancoulier.

Physalis peruviana L. Solanacées, groseiller canaque ou coqueret péruvien.

n. d'un clone de Dioscorea alata, igname.

Sonchus oleraceus L. Composées, laiteron.

Mariscus pennatus Schinz et Guill. Cypéracées, roseau d'eau de Nouvelle-Calédonie.

Miscanthus japonicus Anders. Graminées, roseau du Japon.

Pyrrhosia confluens Ching. (= Cyclophorus confluens Christ.).

Microsemma salicifolia Labill. Ternstremiacées.

n. d'un clone de Colocasium antiquorum, taro.

Myoporum salicifolium ( $=M$. cuneifolium $\mathrm{Kranz}$, $=\boldsymbol{M}$. crasifolium Forst). Myoporacées, faux santal.

Myoporum tubiflorum (=M. tenuifolium Forst.). Myoporacées, grande citronnelle.

Hemicyclia Leplanchei Baill. Euphorbiacées.

$=$ l'herbe marine, Potamogetanacées et Hydrocaritacées marines.

Cynodon dactylon (L.) Pers. Graminées, chiendent-pied de poule, Bermuda grass.

Cryptocarya lifuensis. Lauracées, fausse citronnelle.

Eragrostis pilosa Beauvois (=E. diandra Stend.). Graminées, herbe-à-griffes.

Homalanthus nutans Forst. Pax (= Croton nutans Vahl. Euphorbiacées. 
Hapetra ne hnagejë, cf. Kakejë

Hapice, ef. Sejë

\section{Henedrai \\ Henefini \\ Heo \\ Honasez, Holasez \\ Honasez ne hun 'étë, cf. Sezinaea}

Hödiny

Hû

Hâ (Hue)

Hûje madra

Hâje wië
Huni
Huo
Hla

Hlo gutu

Hmacatresi

Hmalakap

Hmalap

Hmana

Hmehlöm

Hmejex

Hmejieng

Hmelea

Hmelexeci

Hmenë
Lotus australis var. Andr. (= L. anfractuosus Baker). Légumineuses.

Portulaca oleracea L. Aizoacées, pourpier commun, pigweed, purslane.

Sarcostemma australis $\mathrm{R}$. Br. Asclepiadacées.

n. d'un clone de Dioscorea alata, igname.

n. d'un clone de Dioscorea alata, igname.

Wedelia biflora DC. Composées, faux topinambour, herbe à cochons.

Wedelia uniflora D.C. Composées, faux topinambour de rocaille.

Pittosporum spp. lifuense $(=P$. suberosum $\mathrm{Br}$. et Gris $=P$. obovatum Guill.). Pittosporacées. Ixora cauliflora Montr. Rubiacées.

Ficus leiocarpa Bur. Moracées.

1) Zebrina pendula Schnizl (Tradescentia pendula Schnol.). Commelinacées, petite misère longue.

2) Rhaeo discolor, grande misère rouge.

Commelina nudiflora. Commelicacées.

Clematis glycinoides DC. Renonculacées, clématite. Codiaeum inophyllum Müll. Arg. Euphorbiacées, bois de sang.

Musa sp. Musacées.

Claoxylon insularum Müll. Arg. Euphorbiacées.

Fontainea pancheri Heckel. Euphorbiacées, baloghia.

Desoxylum bijugum Scemann (D. albicans Wieill. $=D$. lessertianum Berth.). Meliacées, arbre à sagaies.

Ficus prolixa Forst. Moracées, banyan.

Cryptocarya elliptica Schl. Lauracées, fausse citronnelle ou faux santal.

Maba fasciculosa F. V. Müll. Ebenacées.

Ficus habrophylla var. Moracées, figuier à figue verte.

Ochrosia elliptica (Labill.) Hensl : (= Bleekeria elliptica Koidz = Ochrosia oppositifolia K. Schum $=O$. parviflora (Forster) Henslow). Apocynacées, faux caféier.

Psychotria collina Labill. Rubiacées.

Glochidium glaucum $(\boldsymbol{G}$. caledonicum Baill. $=\boldsymbol{G}$. diospyroides Schl.). Euphorbiacées. 
Hmenepë

Hmetreun

\section{Hmetrewen}

\section{Hmitre \\ Hmitre ixeg \\ Hmitre $i$ papale \\ Hmitre ka cat \\ Hmitre madra, cf. Hmitre ixeg \\ Hmitre ne gejë \\ Hmitre ne mëk \\ Hmitre vië \\ Hmö}

Hmu

\section{Hmucici \\ Hmuhmuzë}

Hmuixetë

Hnë
Glochidium glaucum ( $=G$, caledonicum Baill. = G. diospyroides Schl.). Euphorbiacées.

Musa paradisiaca subsp. sapientum L. Musacées, Bananier.

Elaeocarpus persicaefolius Br. et Gris. Elacocarpacées, cerisier bleu ou cerisier à graines bleues.

Plectranthus parviflorus Henck. Labiées.

Sida rhombifolia L. Malvacées, herbe à balai, chanvre de Queensland, Paddy Lucerne.

Plectranthus parviflorus Henck. Labiées.

Oldenlandia paniculata L. (= Hedyolis paniculatus). Rubiacées.

Ageratum corryzoides I. Composées, baume.

Plectanthrus forsteri Benth. Labiées.

Ximenia americana L. (=X. elliptica Forst.). Olacacées, prunier de mer.

Acacia spirorbis Labill. Légumineuses, faux gaïac ou gaïac de Nouvelle-Calédonie.

2 variétés : Hmu madra, faux gaïac rouge. Hmu wië, faux gaïac blanc.

Champignon.

nom d'un clone de Saccharum officinarum, canne à sucre.

Avicennia officinalis L. Verbenacées (gaïac de poule sultane), palétuvier blanc.

Podonephelium Homei Radl (=P. Leplanchi Baill. $=P$. slipitalum Baill. = Cupania stipitata Pauch et Seb, $=C$. erioglossoides Panch $=$ Ratonia Homei Seem). Sapindacées, faux chêne tigré ou pommaderis-ail.

Arytera arcuata Radlk ( $=A$. Collina, $=A$. littoralis B.L.). Sapindacées, chêne-banyan.

Sarcostema australis $\mathrm{R}$. Br. Asclepiadacées.

Pseudomorus brunoniana Endl. Moracées.

Croton insulare Baill. Euphorbiacées, croton.

Scaevola frutescens Krause. Goodeniacées, bóto des malais.

2 variétés : Hnejixetre madra

Hnejixetre wië 
Hno

Hno $i$ xetë

Hngöl, cf. Potre ne Kejë

Hnyim

Hnyimesa

Ifit, ef. Fit

Ig

Ihe $i$ Wananathin, cf. enaji wananathin

Ijim

Ijineth

Ilemen

Inagaj

Inesi

Ipepa

Ipic

Itinege

Iwanamano

Ixe ë

Ixeë ne hnit, cf. Eköhaitr.
Musa oleracea Baker. Musacées.

Calanthe veratrifolia. Orchidacées (i.e. bananier de poule sultane).

Bikkia comptonii. S. Moore. Rubiacées.

Alyxia brevipes Schlechter. Apocynacées, pulasari d'Indonésie.

Melodinus scandens Forst. Apocynacées.

Calophyllum inophyllum L. Guttiferacées, tamanou, Alexandrin laurel, Laurier d'Alexandrie (le fruit $=$ wenefit).

Rivina humilis L. Phytolaccacées.

Lycopodium phlegmaria. Lycopodiacées, cheveux de sorcière, galons-de-gendarme, épaulettesd'officier.

cf. jim. Euphorbia prostrata Ait. Euphorbiacées, rougette.

cf. jineth. Cudrania javanensis Trec. Moracées (le fruit $=$ wenejineth) .

ef. lemen. Citrus limon Burmann $(=C$. vulgaris $=C$. medica, var. limon). Rubiacées, citronnier (le fruit $=$ wenelemen $)$.

Colocasia antiquorum (L.) Schott (=C. esculenta Schott). Aracacées, taro.

n. d'un clone de Colocasia antiquorum, taro.

ef. pepa. Schinus terebenthifolius Raddl. Anacardiacées, faux poivrier (le fruit $=$ wenepepa).

Citrus sinensis Osbeck $(=C$. aurantium L.) var. sinensis L.). Rubiacées, oranger doux (le fruit = wenepic).

cf. Ithenge. Geijera balansae (Baillon) Schinz (= Zantoxylum balansa Baill.). Rutacées.

1. Gossypium barbadense L. Malvacées, cotonnier.

2. Ceiba pentandra (L.). Gaerta (Eriodendron anfractuosum DC.). Malvacées, kapokier.

Jambosa malaccensis (Brong. et Gris) Vieill. (Eugenia Blackenridgei) (ei $\mathrm{Br}$. et Gris $=E$. pseudo-malaccensis, Syzygiuma malaccense Merr.). Myrtacées, pommier canaque, malayapple (le fruit : wenexee, pomme canaque).

Syzygium pseudopinnatum $(=S$. verrucosum Dän). Myrtacées (i.e., pommier canaque de brousse). 
Jaea

Jedr

Jehe

Jehegöti

Jel

Jelep ( $=$ ji helep)

Jeni

Jenij

Jepujen

Jez

Ji

Ji utine kejë

Jië, ef. Omina

$\boldsymbol{J i j i}$

Jilig

Jineth

Judreli

Juipeng

Kacatr,

Kaen

cf. Hmitre Kacat

Kahaitr, ef. wecia

Kahmed

Kakejë, cf. Hnapetra ne hnagejë

Kakötr n. d'un clone de Musa sapientum, bananier.

Rottboellia coelorachis Forst. Graminées.

Rapanea lecardii Mez. Myrsinacées.

Rapanea citrifolia Mez $(=R$. macrophylla Men. $=R$. lifuensis).

Derris uliginosa (Will.) Benth. $(=D$. trifoliata Lour. = D. heterophylla Backer). Légumineuses, túba laut des malais.

n. d'un clone de Cordyline terminalis (L.) Kunth (= Taetsia fructicosa Merr.). Liliacées, «ti» rouge ou \& $\mathrm{ti}$ » de brousse, dracoena de brousse.

Maesa novo caledonica Mez. (= Rapanaea novocaledonica). Myrsinacées, liane à eau.

Dianella intermedia. Liliacées.

n. d'un clone de Dioscorea alata, igname.

Imperata cylindrica Beam. Graminées, diss.

Cordyline terminalis Kunth $(=$ Taetsia fructicosa Merr. $=T$. neo-caledonica). Liliacées, Dracena vert, $\mathrm{ti}$.

n. d'un clone de Cordyline terminalis.

Cordia Myxa L. (= Cordia obliqua Willd.). Cordiacées, Cunoniacées, gommier.

Harpullia austro-caledonica Baill. Sapindacées, chêne blanc.

Bidens pilosa. Composées.

1. Jilig foë, Bidens sp.

2. Jilig trahmany, Bidens pilosa, beggar-ticks.

Cudrania javanensis Trec. Moracées.

Tithonia diversifolia (Hemsl) A. Gray. Composées, soleil, marguerite, tournesol.

Champignon comestible.

Solanum vieillardii. Solanacées.

Sida rhombifolia L. Malvacées, Herbe à balai, chanvre de Queensland, Paddy Lucerne.

Intzia bijuga (= Afzelia bijuga). Légumineuses, Kohu.

Alocasia macrorhiza (L.) Schott. Aracacées, taro sauvage.

n. d'un clone de Colocasia antiquorum, taro.

Lotus australis Andr. Légumineuses.

n. d'un clone de Musa sapientum, bananier. 
Kapalulu, cf. Atremele ka palulu

\section{Kapes}

\section{Kapi \\ Kasitapa \\ Kecedan, \\ Ket

$$
\text { cf. Hnëthith }
$$

\section{Ketinajo}

\section{Keti ne hnaop \\ Keti ne hnil \\ Keti ne sinöe \\ Kiamu \\ Kit}

\section{Kofi ne knit, cf. Tixeitr \\ Koko \\ Koko etha \\ Ködra \\ Kuka (Wet), cf. Gem (Lôsi)}

\section{Kumala}

Lali, cf. Penekulali

\section{Lamano}

Lapiny

Latana

Le

Lili

Lueihlo

Lupias
Graptophyllum pictum. Acanthacées.

Brassica oleracea L. var. capitata L. Crucifères, Choux-pomme.

Salvia occidentalis Schwarz, Labiées, sauge bleue. Anona squamosa L. Anonacées, pommier cannelle, Custard apple.

Arytera arcuata Rdlk. (A. collina $=A$. littoralis B. L.). Sapindacées, Chêne-banyan.

Cyclosorus invisus Cop. (= Dryopteris invisa Forst.) Fougères, Polypodiacées.

1. Dryopteris immersa O. Ktze (= Lastraea immersa Forst). Polypodiacées.

2. Pteris vittata L. Polypodiacées.

Asplenium sp. Polypodiacées.

Asplenium cuneatum Lam. Polypodiacées.

Nephrolepsis exaltata L. Polypodiacées.

n. d'un clone de Musa sapientum, bananier.

Stachytarpheta indica L. Vahl = Stachytarpheta jamaicensis Vahl). Verbenacées, herbe bleue, verveine bleue.

Chomelia truncatocalyx Guill. $(=C h$. lifuana Dän). Rubiacées, caféier de brousse, faux caféier de Lifou.

Dioscorea alata L. Dioscoreacées. Igname, Yam.

n. d'un clone de Dioscorea alata, igname.

Mucuna gigantea (Willd.) DC. (= Dolichos giganteus Willd.). Légumineuses (le fruit : wenegem = haricot géant).

Ipomaea batalas L. Convolvulacées, patate douce.

n. d'un clone de Dioscorea alata, igname.

n. d'un clone de Musa sapientum, bananier.

n. d'un clone de Dioscorea alata, igname.

Lantana camara L. Verbenacées, lantana.

Stephania Forsteri, Asa Gray. Menispermacées.

1. Crinum asiaticum L. ainsi que genre Chira et Nerine. Amaryllidacées, lis.

2. Zephyrantes rosea. Amaryllidacées, petit amaryllis des prés, petit lis rose.

n. d'un clone de Musa sapientum, bananier.

n. d'un clone de Dioscorea alata, igname. 
Macaté

\section{Madra \\ Madra hni \\ Madra thöni \\ Maea \\ Maefedr \\ Maga \\ Makadrë}

Mako

Makue

Malacatr

Malo

Maniapo

Maniota

\section{Matranidrön}

Manv

Meci

Mecipë

Medreth

Medrudro

Megel

Meigöt

Mëken

Melo

Mesiahot

Mesup
Terminalia catappa L. Combretacées. Badamier ou Amandier de l'Inde, Indian Almond.

Balogluia lucida Endl. Euphorbiacées, bois de sang.

n. d'un clone de Colocasium antiquorum, taro.

Bischoffia javanica. Euphorbiacées.

Coleus scutellarioides Benth. Labiacées.

Stenolobium stans. Bignoniacées.

Mangifera indica L. Anacardiacées, manguier.

Nom d'un clone de Dioscorea bulbifera, patate aérienne.

Elattostachys apetala Radlk, var. robustior. Sapindacées, faux chêne blanc.

n. d'un clone de Colocasium antiquorum, taro.

Phyllanthus simplex var. pratense Retz. (= Ph. virgatus Font.). Euphorbiacées.

Epipremnum pinnatum Engl. Aracacées.

Carica papaya I. Caricacées, Papayer.

1. Manihot utilissima Pohl $(=M$. dulcis Baille $=M$. esculente Kranz). Euphorbiacées, manioc.

2. n. d'un clone de Dioscorea alata, igname.

Bryophyllum Calycinum L. Crassulacées, \& feuille multipliante $\gg$.

Rhamnella vitiensis Benth. (= Berchemia fournieri Panch). Rhamnacées, Rhamnelle de Fiji.

Euroschinus obtusiolius Engl. Anacardiacées, térébenthine de Lifou.

Dysoxylum bijugum. Méliacées.

Eugenia mendute Guill., Eugenia uniflora L. Myrtacées, cerisier de Cayenne.

Canarium balansae. Burseracées, amandier de Java, noix-mission.

Garcinia pedicellata Seem $(=$ G. mangotia Deplanche ex-Pierre $=$ Clusianthemum pedicellatum Vieill. $=C l$. Ceniaceum Vicill.). Guttifères, prunier canaque.

n. d'un clone de Musa sapientum, bananier.

Cucumis melo L. $(=C$. acidus Jacq. $=C$. aspera Forst. $=$ C. pubescens Willd.). Cucurbitacées, melon.

Marsdenia sp. Asclepiadacées.

Pichonia balansana Pierre (= Iucuna balansana). Sapotacées. 
Meiz, cf. Watolea

Mik, ef. Wenek

Mimij

Mino

Miny

Momo

Muni

Naina, cf. Bona

Nemuni
Nihlë
Nin
Nu
Nu hatro
Nu tehle
Numea
Nyinyitr
Oriadra
Omina, cf. Jië
Otr, cf. Axöti ne hnit
Otë, cf. Wathangöni
Oxelek

Oleng

On

\section{Pahatr}

Pahatre ne sinoë

Pahatre nyine xen
Zea mays L. Graminées, maïs.

Flagellaria neo-caledonica Schltr. Flagellariacées. Malaisia tortuosa Blanco var. $\mathrm{Fl}$. filip viridescens Bur. Moracées.

Pandanus (cf. P. tectorius) Vieillardii Martelli $(=P$. fragrans Gaud $=P$. pedonculatus $\mathrm{R}$. et Br.). Pandanacées, pandanus du bord de mer. n. d'un clone de Musa sapientum, bananier.

Semecarpus atra Vieill. Anacardiacées, muni madra, var. rouge, muni wië, var. blanche, pommier-cajou, pommier d'acajou, goudronnier.

Cajanus pseudocajan Schnz (=C. indicus Spreng). Légumineuses, ambrevade, pois d'Angole.

n. d'un clone de Dioscorea alata, igname.

Secamone insularis Schlechter. Asclepiadacées.

n. d'un clone de Saccharum officinarum, canne à sucre.

Cocos nucifera. Palmacées, cocotier.

Microkentia sp. Palmacées.

Basselinia sp. Palmacées.

n. d'un clone de Dioscorea alata, igname.

n. d'un elone deDioscorea alata, igname.

Nerium oleander L. Apocynacées, laurier rose.

Cordia myxa Roxb. Cordiacées, gommier.

Pseudomorus bruoniana Bur. Moracées.

Lysimachia mauritiana Lam. Primulacées, lysimaque.

Morinda citrifolia L. Rubiacées, fromager, mûrier indien, canary wood.

Diospyros Olen Hiern ( $=D$. vitiensis Gillespie). Ebénacées, ébénier blanc, plaqueminier, kaki.

Artocarpus communis Forst. (=A. incisa L. = A. altilis (Park) Forsberg). Moracées, arbre à pain.

Microserium punctatum Cop. Schwartz $(=$ Polypodium punctatum). Polypodiacées.

Psilotum nudum Sch. Polypodiacées.

Asplenium nidus L. Polypodiacées, Fougère, nid d'oiseau, langue de chat, birdnest fern. 
Paniapo

Paliat

Panganai (Lösi), ef. $\mathrm{Ai}$ (Wet)

Papua

Paxanutr

Paza

Pasa madra

Pedehe

Pehna

Pelausia

Peledrë

Pen

Penekulali, ef. Lali Pepa

\section{Pepethua}

Petisenven

Petipet

$\boldsymbol{P i}$

\section{Piagot \\ Pihla \\ Piliwe \\ Pô}

Pô

Pohlë

Poisin

Pojë
Ananas comosus (L.) Merrill. Bromeliacées, ananas.

Vernonia cinerea. Composées.

Geitonoplesium cymosum (R. Br.) A. Lum. Liliacée.s

n. d'un clone de Dioscorea alata, igname.

Thieghemopanax sessiliflorus Rvig (Th. austro calëdonicus R. V.).

Hibiscus tiliaceus var. edulis $(=H$. Paritium Wight et Arn.). Malvacées, bourao comestible, cotton tree.

Hibiscus tiliaceus. Malvacées, bourao rouge, bourao des marais.

n. d'un clone de Dioscorea alata, igname.

Parasponia Andersonii Planch. Ulmacées.

Sida acuta Bur. Malvacées, parousie.

1. Hibiscus rosa-sinensis Linn. Malvacées, hibiscus.

2. Hibiscus rosa-sinensis var. schizopetalus Hook. f. Malvacées, hibiscus.

Ischaemum muticum L. Graminées, rumpetkerumpet des malais.

n. d'un clone de Dioscorea alala, igname.

Schinus terebinthifolius Raddi. Anacardiacées, faux poivrier.

Cesalpinia crista L. Légumineuses.

Cenchrus calyculatus Cav. Graminées.

Sesuvium portulacastrum L. Aizoacées, pourpier sauvage.

Glycine tabacina (Labill) Benth. (= Kennedia tabacina Labill.). Légumineuses.

n. d'un clone de Dioscorea alata, igname.

n. d'un clone de Dioscorea alata, igname.

n. d'un clone de Dioscorea alata, igname.

Montrouziera cauliflora Panch. et Triana. Guttifères, arbre à bois jaune, houp.

n. d'un clone de Musa pasientum, bananier.

Clerodendron inerme (L.) Gärtn. Verbenacées, Pohlë madra; variété rouge; Pohlë wië: variété blanche.

Catharanthus roseus. Apocynacées.

Achronychia laevis Forst. Rutacées. 
Potr, poc

Potre ne kejë, Pö ef. Hngöl

Puifelö, cf. Puisela

Puisela, ef. Puifelö Pulipul

Qajaatr

Qaninewe

Qateiaji

Qaqa

Qatrë

Qea

Qeihnitr

Qeleleny

$\boldsymbol{S a}$

Salali, cf. Silali. Weë

Salatr

Sedr

Segöl

Sehngödr

Sejë, cf. Hapice

Selek

Sere

Seshë

Seunöz

Seuseu
Fagrea Schlechterii Gild et Bened. (F. obovata). Logoniacées, bois à tabou, bois pétiole, bijouen. Bikkia comptonii Modre. Rubiacées.

Manilkara Pancheri Pierre (= Mimusops Pancheri Baill.). Sapotacées, chêne-tann, buni.

Murraya crenulata DC. Rutacées (= Chalcas crenulata), mock orange.

Murraya crenulata DC. Rutacées.

Geniostoma foetens Baill. (G. rupestre Harvard). Logoniacées.

Polyaltia nitidissima. Anonacées.

Plumbago zeylanica L. Plumbaginacées.

Hydrocotyle asiatica L. (= Centella asiatica Urban). Ombellifères.

Grewia crenata Forst. Tiliacées.

n. d'un clone de Saccharum officinarum, canne à sucre.

Bryonopsis affinis Cogn. Cucurbitacées.

Olea thozetii Pancher et Sebert. Oléacacées, Olivier de Nouvelle-Calédonie.

Polyscias pinnata Forst. Araliacées, manguette.

Ficus microcarpa var. hilii Corn $(=F$. schlechteri). Moracées.

Eugenia oraria Guill. Myrtacées.

Allium tuberosum Rottler. Liliacées, petit oignon vert, kuchai de Malaisie.

Tribulus cistoides ( $T$. moluccanus). Zygophyllacées.

Smilax purpurata Forst (S. plurifurcata). Liliacées, salsepareille.

Phyllantus urinaria L. Euphorbiacées.

Portulacca oleracea L. Aizoicées, pourpier commun, pigweed, purslane.

Amarantus gracilis Desf. (= A. curentus var. paniculatus Thel.). Amarantacées.

Phaseolus sp. Légumineuses, haricot.

Sida nummularia E. G. Backer $\mathrm{f}$. Malvacées.

Piper austro-caledonicum DC. Pipéracées.

n. d'un clone de Dioscorea alata, igname. 
Sez, cf. Sezijat

\section{Sezijat}

Sezinaea, cf. Honaseze nehun'ete

Si, ef. Hmejieng

Sidrë

$S i j$

Silali, ef. Salali. Weë Sinegit

Sitrae

So $=$ Sho

Sojel $=$ Shojel

Soletë $=$ Sholetë

Soma $=$ Shoma

Somadra $=$ Shomadra

$\boldsymbol{S} \ddot{\boldsymbol{o}}$

Sö ne gejë

Sumaleng

Taisepu

Taisepu ne hnaop

Tana

Tapakai

Tasuo

Tehmex

Telewegeth

Tilö

Tim
Randia sezital (= Gardenia sezitat Baill.). Rubiacées.

cf. Sez.

Vedelia uniflora DC. Composées, faux topinambour, herbe à cochons.

Ficus habrophylla. Moracées, figuier à figue verte.

n. d'un clone de Saccharum officinarum, canne à sucre.

Cupaniopsis glomerifera Radlk. Sapindacées, Chêne-blanc.

Eugenia oraria Guill. Myrtacées.

Tectaria lifuensis Cop. $(=T$. latifolia Forst.). Polypodiacées.

Melia azedarach L. Meliacées, lilas de Perse.

Cerbera manghas I. Apocynacées, manguiercerbera, faux manguier.

Euphorbia kanalensis Boiss. Euphorbiacées.

Loranthus. Loranthacées.

Codiaeum variegatum Blume. Euphorbiacées, croton ornemental.

Cerbera manghas L. var. rouge. Aponynacées.

Aglaia eleagnoides (A. Jus.) Benth. Meliacées, bois-rose, petit milnea.

Allophyllus ternatus Radlk (A. timorensis (DC.) Blume, = A. cobbe BI.). Sapindacées, faux kohu.

Desmodium adscendens. Légumineuses.

Tephrosia purpurea Pers. $(=T$. piscatoria (Ait.) Pers.). Légumineuses.

Asplenium falcatum L. Polypodiacées.

n. d'un clone de Dioscorea alata, igname.

Santalum austro-caledonicum Vieill. Santalacées, santal.

n. d'un clone de Dioscorea alata, igname.

Boerhavia repens $\mathrm{L}$. $(=B$. diffusa $\mathrm{L} .=B$. carunculata Baill.). Euphorbiacées.

Minusops parvifolia (R. Br.) $(=M$. melangi L. var. parvifolia). Sapotacées, raporé.

Lepironia mucronata Cypéracées, jonc.

Passiflora suberosa L. var. viridis. Passifloracées, pomme-liane vert violacé. 
Tinege, cf. Itinege, Ithenge

Tixeitr, cf. Kofi ne hnit

\section{Titajidr}

Tö

$\boldsymbol{T} \boldsymbol{u}$

Tuhao

The

Theletha, ef. Wenepale

Thepë

Thëtesi

Thewek

Thidra

Thidrahnit, Thidranehnit

Thidraxep

Thili

Thuahaik

Thuipel

Thulumat

Thulumate vië

Thulumate madra

Thumulati ka ngongo Thuma

Thuma hait

Thuma koko

Thuma madra

Thuma wautivelewel
Geijera Balansae Schinz. Rutacées.

Chomelia truncatocalyx Guil. Rubiacées $(=\mathrm{Ch}$. lifuana Dän.), i.e. caféier de brousse, faux caféier de Lifou.

n, d'un clone de Musa sapientum, bananier.

Lippia nodiflora Rich. Michx. Verbenacées.

Solanum nigrum L. Solanacées, brède-morelle, morelle noire.

Solanum viride $\mathrm{R}$. $\mathrm{Br}$. Solanacées, morelle blanche, épinard chinois.

Serianthes calycina Benth. Légumineuses, acacia failfail.

Dolichos lablab (L. sp.) Linné. Légumineuses, haricot canaque, dolique d'Egypte, lablab, hyacinth bean, bátao.

Melochia odorata L. f. Sterculiacées.

(= The atesi). Guetlardia speciosa L. Rubiacées, faux figuier.

Zingiber zerumbet Sm. Zingibéracées, gingembre, ginseng.

Achyranthus aspera L. Amarantacées.

1. Thidra föe

2. Thidra trahmany

Elaeodendron sp. Celastracées, bois-jaune.

Elaeodendron curtipendulum. Celastracées, boisjaune.

Hoya neo-caledonica Schlecht. Asclepiadacées.

Wickstroenia viridiflora Meissn. Thymeléacées.

Solanum vieillardii Bit. Solanacées.

Acalypha neo-caledonica Müll. Arg. Euphorbiacées.

var. blanche.

Acalypha grandis Benth. (= Caudafelis agrostis Rumphius). Euphorbiacées.

Acalypha pancheriana. Euphorbiacées.

Dioscorea bulbifera L. Dioscoreacées, ignamepatate, patate aérienne, soa.

n. d'un clone de Dioscorea bulbifera, thuma amer.

n. d'un clone de Dioscorea bulbifera.

n. d'un clone de Dioscorea bulbifera, thuma rouge.

n. d'un clone de Dioscorea bulbifera. 
Thuma wië

Thumeny

Trakala

Tresi

Trineng

Truhe

Trudrö

$\boldsymbol{U a}$

Ug

Ujahao

$\boldsymbol{U} \boldsymbol{k}$

Ulë

Umeci

Ura

Utem, cf. Uxapo

Utr

Uxapo, cf. Utem

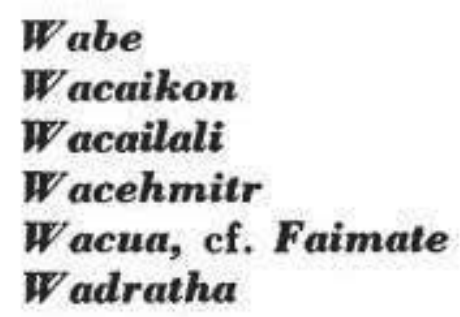

Wadratha

Waël

Waëlebic

Wafel

Wagelava n. d'un clone de Dioscorea bulbifera, thuma blanc. Euphorbia pancheri Baill. Euphorbiacées. Celtis conferta Planchon. Ulmacées.

Barringtonia asiatica (L.) Kurz $(=B$. edulis Seem). Barringtoniacées, noyer-navelé (le fruit: wenetresi).

Zieridium pseudo-obtusifolium Guill. (= Evodia pseudo obtusifolia. Rutacées.

n. d'un clone de Dioscorea alata, igname.

Messerschmidia argentea (L.) Johnston (Tournefortia argentea $=$ Heliotropium argenteum). Borraginacées, herbe à gratte, faux tabac, herbe à Calimbre.

Microstylis taurina Reichb. f. Orchidacées.

Gardenia Urvillei Montr. Rubiacées, Gardenia de Dumont d'Urville.

Desdemodium umbellatum (L.) DC. Légumineuses.

Nephrolepsis hirsutula Presl. Polypodiacées.

Erigeron crispus Pour. Composées.

Crinum pedunculatum $\mathrm{R}$. Br. Amaryllidacées, lis de forêt.

n. d'un clone de Colocasia antiquorum, taro.

Canavalia obtresifolia DC. (C. rosea DC. $=C$ lineata Prain. = Caraca littorea Rumphius). Légumineuses.

n. d'un clone de Dioscorea alata, igname.

Canavalia obtusifolia DC. Légumineuses.

n. d'un clone de Dioscorea alata, igname.

n. d'un clone de Dioscorea alata, igname.

Balanophora fungosa Forster. Balanophoracées. Davallia solida (Forst.) Schwartz. Polypodiacées. Ficus edulis. Moracées, figuier à figue rouge.

Pandanus macrocarpus Vieill. (= Barrotia macrocarpa Brong.). Pandanacées, faux pandanus.

n. d'un clone de Dioscorea alata, igname.

n. d'un clone de Dioscorea alata, igname.

n. d'un clone de Dioscorea alata, igname.

Jatropha curcas L. Euphorbiacées, pignon d'Inde, pourghère.

n. d'un clone de Dioscorea alata, igname. 
Wahana, ef. Wakana

Wahana $i$ wananathin

Wahla

Wahle köti

Wahlep, ef. Wameze

Wahnyaöl

Waijij

Wailatha, cf. Thuma

Withiedr

Wakalua

Wakana, ef. vahana

Wakana $i$ Wananathin

Walei

\section{Walei kau} (waré des vaches)

Walei waël (waré sauvage)

Wameze, of. Wahlep Wanaithihlë

Wanemana
Wanepi
Wanauëng
Wapiopio
Was
Wasuma
Watolea, cf. Meiz
Watoma

Watu
Cissus glaucorama Planch. Vitacées.

Cayratia japonica Gagnep. Vitacées, vigne de sorcière.

n. d'un clone de Dioscorea alata, igname.

Phymalodes scolopendra (Burm. et Schirm) Cheng $=$ Polypodium scolopendria Burm. $=$ P. phymatoides L. = Microsorium scolopendrium Cop.). Polypodiacées, Fougère scolopendre.

Hemigraphis replans T. Anders. Acanthacées.

Ioiscorea pentaphylla L. Dioscoreacées.

Pisonia aculeata L. Nyctaginacées, pisonie épineux de Pondichéry.

Dioscorea bulbifera. Dioscoreacées, patate-igname, patate aérienne, soa.

Mallotus repandus var. scabrifolia Müll. Arg. (= Mappa scandens Panch). Euphorbiacées.

Cissus glaucorama Planch. Vitacées.

Cayjratia japonica Gagnep, Vitacées, vigne de sorcière.

Dioscorea esculenla (Lour.) Burk. Dioscoreacées, Waré.

n. d'un elone de Dioscorea esculenta.

n. d'un clone de Dioscorea esculenta.

Hemigraphis reptans T. Anders. Acanthacées.

Ipomoea angulala $(=I$. obscura, Ker Gaul. =

1. coccimea L. $=$ I. Qamoclit $\mathrm{L} .=$ Qamoclit cocinea Moench $=I$. congesta $\mathrm{R}$. Br.). (onvolvulacées, liseron écarlate.

n. d'un clone de Dioscorea alata, patate.

n. d'un elone de Dioscorea alata, patate.

Spinnifex hirsulus Labill. Graminées, Spring rolling grass.

n. d'un clone de Dioscorea alata, igname.

n. d'un clone de Colocasium antiquorum, taro.

Zea mays. Graminées, maïs.

Ryssopteris discolor G. Dr. $(=R$. timorensis B1. $=R$. austrocaledonica Ndzu). Malpighiacées.

Lycopersicum esculentum Mill. Solanacées, tomate. 
Wathangöni, ef. Otë

Wathanej,
cf. Hmitre ne geje
Wathë
Wan
We
Wecia, cf. Kakaitr
Weco
Wedr
Wë, cf. Silali
Weg

\section{Wegelëtë}

Wej

\section{Wejihnit, cf. Wejingongo

Weketh
Wel
Weliwel
Wenefela
Wenenu

Wenek, ef. Mik

Wenepapale, cf. Theletha Wenewej

Wenewej

Weve

Wewej

Wexu
Lysimachia mauriliana Lam. Primulacées, Lysimaque.

Triumphelta procumbens Forst. Tiliacées, Rubili des malais.

Broussonetia papyfera Ventena. Moracées, papyrus.

Corynocarpus dissimilis Hemsl. Corynocarpacées.

n. d'un clone de Saccharium officinarum, canne à sucre.

n. d'un clone de Colocasium anliquorum, taro.

n. d'un clone de Musa sapientum, bananier.

Pandanus tectorius Sol. (=P. odoratissimus I..). Pandanacées, pandanus textile, screw pine.

Eugenia oraria Guill. Myrtacées.

Dodonaea viscosa (L.) Jaq. Sapindacées, reinette de la Réunion.

n. d'un clone de Saccharum officinarum, canne à sucre.

Colocasium sp. Aracacées, taro-épinard.

Weji madra, à pétiole rouge.

Weji wië, à pétiole blanc.

Hibiscus manihol I. (= Abelmoschus manihot Medik, Hibiscus abelmoschus). Malvacées, ambrette.

Carissa lucidula Sleumer. Apocynacées.

Jacquemontia paniculata Hal. Convolvulacées.

Plerocaulon redolens F. Vill. Composées.

n. d'une clone de Dioscorea alata, igname.

noix de coco.

wenenu madra, noix à cosse rouge.

wenenu wï̈, noix à cosse verte.

Flagellaria neo-caledonica Schltr. Flagellariacées.

Dolichos lablab (I. sp.) I. Légumineuses, haricot canaque, dolique d'Egypte, lablab.

Tacca pennatifida Forst. ( $=T$. leontopetaloides O. K.). Taceacées, A rrow-root de Tahiti, pia.

n. d'un clone de Dioscorea alata, igname.

(Chrosia lifuana D. $(=O$. thiollieri Montr.). Apocynacées, faux caféier de Lifou.

Adiantum capillus veneris $\mathbf{L}$. Polypodiacées, fougère choveux de Vénus.

Wexu foë. Jasminum elatum Panch. Jasmin. 
Wia

Wia madra

Xaca, ef. Al.

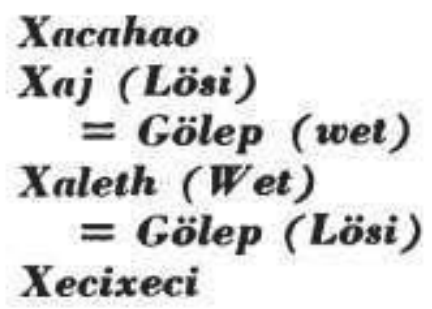

Xeё, cf. Ixeё

Xeutr
Xejima
Xenixenit
Xenyë
Xenyë pupu
Xetr
Xotha hao,
cf. Ageti temoni
Xölëny

Ẍ̈lep

$X \boldsymbol{u}$, cf. $\boldsymbol{P} \ddot{\boldsymbol{o}}$

Xuluhnep

Xutrepet

Xutrepet

Xuxutrotro
Wexu trahmany. Jasminum leratii. Oleacées, Jasmin.

Saccharum officinarum I. Graminées, canne à sucre.

n. d'un clone de Saccharum officinarum, canne à sucre rouge.

Premma sambucina (=P. integrifolia) (L.) Mantoue. Verbenacées.

Colubrina asiatica (L.) Brongn. Rhamnacées.

Schefflera golip Baill. Araliacées, golip blanc à pétiole vert, ralia.

Octotheca ouveana (Dän.) Guill. (= Dizygotheca ouveana Dän.). Araliacées. Golip rouge.

n. d'un clone de Saccharum officinarum, canne à sucre.

Jambosa malaccensis (Brong. et Gris) Vieill. = Eugenia psendo malaccensis, $=E$. Blackenridgei Br. et Gr. = Syzygium malaccense). Myrtacées, pommier canaque, malay apple.

Delarbrea collina Vieill. Araliacées.

Triumphetta rhomboïdea Jacq. Tiliacées.

Cassytha filiformis L. Lauracées.

n. d'un champignon comestible.

n. d'un champignon phosphorescent.

Maba buxifolia Pers. Ebenacées.

Acacia farnesiana (I.) Willd. Iégumineuses, Cassie.

Micromelum pubescens B1. (= M. minutum Wight et Arnott). Rutacées, thé de Lifou.

n. d'un clone de Saccharum officinarum, canne à sucre.

Montrouziera cauliflora Panch. et Triana. Guttifères, houp.

Morinda forsteri. Rubiacées.

Licania lifuana Dän $(=L$. gerontogea Schlechter, $=L$. rhamnoides Guill.). Chrysobalanacées.

n. d'un clone de Saccharum officinarum, canne à sucre.

Crotalaria striata DC. Iégumineuses. 
Zamelo

Zava

Zilo

Ziluth

Zimaca, ef. Ziutr

Ziutr

Zizania
Syzygium lateriflorum Bron. et Gris $(=S$. multipetalum Panch. $=S$. jambolanum, $=$ Eugenia jambolana $\mathrm{DC} .,{ }^{\prime}=E$. pseudomalaccensis Vieill. $=E$. Cumini (L.) Skeels). Myrtacées, jamelonier, jamelonguier.

Vigna sinensis. Légumineuses, haricot de Java, cowpea, pois à vache.

Coix lacryma jobi. Graminées, larmes de Job.

cf. Ziutr

Indigofera suffruticosa Mill. (=I. anil L.). Légumineuses, indigotier. 